\title{
ASSOCIATION BETWEEN VITAMIN D STATUS AND METABOLIC DISORDERS IN PREMENOPAUSAL WOMEN WITH AUTOIMMUNE HYPOTHYROID DISEASE
}

DOI: 10.36740/WLek202107111

\author{
Oksana 0. Chukur, Nadiya V. Pasyechko, Anzhela 0. Bob, Iryna V. Smachylo, Liudmyla V. Radetska \\ HORBACHEVSKY TERNOPIL NATIONAL MEDICAL UNIVERSITY, TERNOPIL, UKRAINE
}

\begin{abstract}
The aim: Is to establish a relationship between serum vitamin D level with carbohydrate and lipid indexes in women with autoimmune hypothyroid disease.

Materials and methods: 146 women with autoimmune hypothyroid disease were examined in the period 2017-2019, who signed the informed consent. The mean age of women was $43.8 \pm 0.7$ years. Anthropometric, general clinical and biochemical examinations were performed including determination of lipid metabolism, hydrocarbon metabolism and establishment of vitamin-D status.

Results: Vitamin D deficiency was found in 78.8\%, insufficiency in $17.1 \%$ of women with autoimmune hypothyroidism. Statistical processing of results was carried out and established strong negative correlation between $25(\mathrm{OH}) \mathrm{D}$ and Thyroid Peroxidase Antibody $(r=-0,77)$ and thyrotropic hormone level $(r=-0.72), p<0,05$. Strong inverse correlations were found between vitamin D levels and body mass index $(r=-0,74)$ and total cholesterol levels $(r=-0,72)$, negative correlation of medium strength was with highdensity lipoprotein cholesterol $(r=-0,58)$, triglycerides $(r=-0,46)$, atherogenic coefficient $(r=-0,65)$ and the HOMA-IR $(r=-0,57)$, The values of $p<0.05$ were considered reliable. Conclusions: The incidence of vitamin D hypovitaminosis in women with autoimmune thyroid disease is significantly higher than in the healthy population. Low vitamin $D$ status is significantly associated with autoimmune thyroid dysfunction and determines the degree of metabolic disorders and cardiovascular risk in premenopausal women with autoimmune hypothyroid disease.
\end{abstract}

KEY WORDS: hypothyroidism, vitamin D, premenopausal age, women

Wiad Lek. 2021;74(7):1612-1616

\section{INTRODUCTION}

Vitamin $\mathrm{D}$ is an important vitamin, required for the regulation of calcium and bone health [1]. Going far beyond its classical role in calcium and bone metabolism, the nonskeletal effects of the importance of vitamin $D$ for various organs and systems have been confirmed, the relationship between its deficiency and various metabolic disorders, diseases of the cardiovascular, endocrine, immune, reproductive and other systems was revealed [2-3]. In Ukraine $81.8 \%$ of adults have an acute deficiency of vitamin $\mathrm{D}, 13.6 \%$ have an insufficient amount, and only $4.6 \%$ of Ukrainians have a sufficient level of vitamin D [4].

Vitamin D deficiency has been found to correlate with an increased incidence of autoimmune diseases, including type 1 diabetes mellitus, rheumatoid arthritis, systemic lupus erythematosus, multiple sclerosis (MS), and Crohn's disease [5-8]. However, it is unclear whether low vitamin D levels closely correlate with the development of autoimmune thyroid disease (AITD) [9].

Thyroid dysfunction is considered as a common endocrine disease and its prevalence has recently increased. This disease affects up to $1.5 \%$ of the population, predominantly females. The prevalence of hypothyroidism (HT) among adult females of different age groups ranges from 3-7.5\% and is more frequent among elderly women [10]. Recent epidemiological studies indicate a relationship between thyroid autoimmunity and vitamin D deficiency [11-13]. Furthermore, the association of lower vitamin D levels with higher prevalence of AITD has been found to be significant in premenopausal, but not in postmenopausal women [14].

Menopausal transition is associated with an increased risk of metabolic syndrome and cardiovascular disease [15]. Metabolic syndrome (MetS) is a condition characterized by the contemporary presence of central obesity, arterial hypertension, altered lipidic and glycide metabolism. Previous reports indicated that increased thyroid-stimulating hormone (TSH) levels within the normal range were significantly correlated with metabolic syndrome parameters, insulin resistance and obesity [16].

Considering the multifactorial development, the high prevalence of hypothyroidism in Ukraine, and the lack of clear recommendations for effective prevention measures, there is a need for a more detailed study of risk factors in the occurrence of this pathology. Although the relationship between Vitamin D and HT has been established long ago, the interaction of thyroid hormone, vitamin $\mathrm{D}$ and its metabolites remains to be unexplored, which confirms the expediency of screening vitamin $\mathrm{D}$ level in patients with autoimmune thyroid disease. 


\section{THE AIM}

To determine the level of vitamin $25(\mathrm{OH}) \mathrm{D} 3$ in premenopausal women with autoimmune pathology of the thyroid gland. Establish a relationship between serum vitamin D level with carbohydrate and lipid indexes in women with autoimmune hypothyroid disease.

\section{MATERIALS AND METHODS}

146 women with autoimmune hypothyroid disease were examined. The study included premenopausal women between the ages of 40 and 54, the mean age was $46,6 \pm 0,8$ years, menopause status was determined using a self-report questionnaire about menstrual bleeding and its regularity. Disease duration was $6.4 \pm 1.7$ (3 to 8 ) years. All participants were on outpatient and inpatient treatment in the department of endocrinology and endocrinological dispensary at the Ternopil University Hospital. Patients with concomitant chronic somatic diseases with severe or progressive course were excluded from the study. All patients signed informed consent to participate in the study. The research was conducted in compliance with all moral and ethical principles according to World Medical Association Declaration of Helsinki, after obtaining the conclusion of the ethics committee at I. Horbachevsky Ternopil National Medical University.

In order to establish or confirm the diagnosis, a general clinical examination, laboratory and instrumental research methods were performed. Anthropometric parameters were measured in all patients and the body mass index (BMI) was calculated according to the formula (mass $(\mathrm{kg}) /$ height $\left.{ }^{2}\left(\mathrm{~m}^{2}\right)\right)$.

To evaluate the functional status of thyroid gland, the level of thyrotropic hormone (TSH), free thyroxine (fT4), free triiodothyronine (fT3) in serum was determined using the electrochemiluminescent method on an automatic analyzer Roche «Cobas-41», via Roshe Diagnostics reagents (Germany), Thyroid Peroxidase Antibody (TPOAb) was determined via Orgentec GmbH kits (Germany).

The levels of total cholesterol (TC), high-density lipoprotein cholesterol (HDLc) and triglycerides (TG) in serum were determined to assess lipid metabolism using automatic biochemical analyzer Roche «Cobas-501» via Roshe Diagnostics reagents (Germany). According to lipidogram indices were determined: low density lipoprotein cholesterol (LDL cholesterol $=\mathrm{TC}-(\mathrm{TG} / 2,2+\mathrm{HDLc}))$ and atherogenic coefficient $(\mathrm{AC}=(\mathrm{TC}-\mathrm{HDLc}) / \mathrm{HDLc})$.

For the study of carbohydrate metabolism, basal glucose level in serum was determined by glucose oxidase method on the automatic biochemical analyzer Roche «Cobas-501» and basal blood insulin level by electrochemiluminescent method using Roche «Cobas-411» analyzer and Roche Diagnostics test systems (Germany).

The HOMA-IR (Homeostasis Model Assessment for Insulin Resistance) index was calculated by the formula: HOMA IR = (fasting glucose $\mathrm{x}$ basal insulin): 22.5.

Determination of level $25(\mathrm{OH}) \mathrm{D}$ in serum was performed in autumn-winter period, by electrochemilumi- nescent method on Elecsys 2010 (Roche Diagnostics, Germany), using the 25-OH Vitamin D ELISA kit (EUROIMMUN, Germany). Vitamin D status was evaluated according to the recommendations of the International Society of Endocrinologists, according to which vitamin $\mathrm{D}$ deficiency is set at a level of $25(\mathrm{OH}) \mathrm{D}$ below $20 \mathrm{ng} /$ $\mathrm{ml}(50 \mathrm{nmol} / \mathrm{l})$, insufficiency of $25(\mathrm{OH}) \mathrm{D}$ in the range of $20-30 \mathrm{ng} / \mathrm{ml}(50-75 \mathrm{nmol} / \mathrm{l})$ and sufficiency of $25(\mathrm{OH}) \mathrm{D}$ above $30 \mathrm{ng} / \mathrm{ml}(75 \mathrm{nmol} / \mathrm{l})$.

Statistical processing of the results and visualization of the obtained data were performed using the statistical analysis package STATISTICA 12. The values of the investigated parameters are given in the form of arithmetic mean values and their mean error $(\mathrm{M} \pm \mathrm{m})$. The probability of difference between the groups was evaluated using the t-test (Student). Correlation analysis and the Pearson linear correlation coefficient were calculated to assess the degree of correlation. The correlation of quantitative indicators was considered as strong at correlation coefficient values from 0.70 to 1.00 , mean strength from 0.30 to 0.69 and weak to 0.29 . The difference between values was considered significant at $\mathrm{p}<0.05$.

\section{RESULTS}

The diagnosis of hypothyroidism was based on characteristic clinical symptoms and hormonal study results. The average level of TSH was $-4,40 \pm 0,21 \mathrm{mIU} / \mathrm{L}$; fT3 $-1.97 \pm 0.09$ $\mathrm{pmol} / \mathrm{L} ; \mathrm{fT} 4-12.96 \pm 0.37 \mathrm{pmol} / \mathrm{L} ; \mathrm{TPOAb}-112.87 \pm 5.61$ $\mathrm{IU} / \mathrm{ml}, \mathrm{p}<0.05$. To compensate for hypothyroidism, patients received levothyroxine sodium in replacement doses ranging from 50 to $150 \mu \mathrm{g}$. According to TSH levels, compensated hypothyroidism was diagnosed in 94 women (64.4\%) and subcompensated in $52(35.6 \%)$. Body mass index (BMI) averaged $29.4 \pm 0,29$ in $49.3 \%$ of the surveyed, which corresponds to overweight; in $44.5 \%$ of women was observed obesity.

The average glucose level reached $5.3 \pm 1.2 \mathrm{mmol} / \mathrm{L}$, which corresponds to the reference values. Basal insulin levels exceeded the control value in $68.2 \%$ of patients, HOMA-IR averaged $3.31 \pm 0.08(\mathrm{p}<0.05)$.

Hypercholesterolemia more than $5.2 \mathrm{mmol} / \mathrm{L}$ was found in $54(26.8 \%)$ of women, HDL cholesterol $<1.15 \mathrm{mmol} / \mathrm{L}$ in $69(48.3 \%)$, LDL cholesterol $>2.6 \mathrm{mmol} / \mathrm{L}$ in $76(75.6 \%)$, $\mathrm{AC}>3$ was observed in $64 \%$ (Table I).

The mean level of $25(\mathrm{OH}) \mathrm{D}$ in women with autoimmune hypothyroid disease was $16.42 \pm 0.57 \mathrm{ng} / \mathrm{ml}$, which corresponds to vitamin D deficiency $(<20 \mathrm{ng} / \mathrm{ml}$ ). (Fig. 1).

Comparative analysis of carbohydrate and lipid metabolism in the study groups showed a significant increase of their levels in vitamin D insufficiency and deficiency patients. In the group of women with vitamin D deficiency were higher BMI, insulin resistance index HOMA and AC in comparison to women who had sufficient level of vitamin D. In the third group, in comparison with the first group, the level of LDLc was twice higher, and the AC was three times higher. Mean LDL cholesterol values were higher by $24.4 \%$, BMI by $42.5 \%$, HOMA insulin resistance index by $52.6 \%$. 


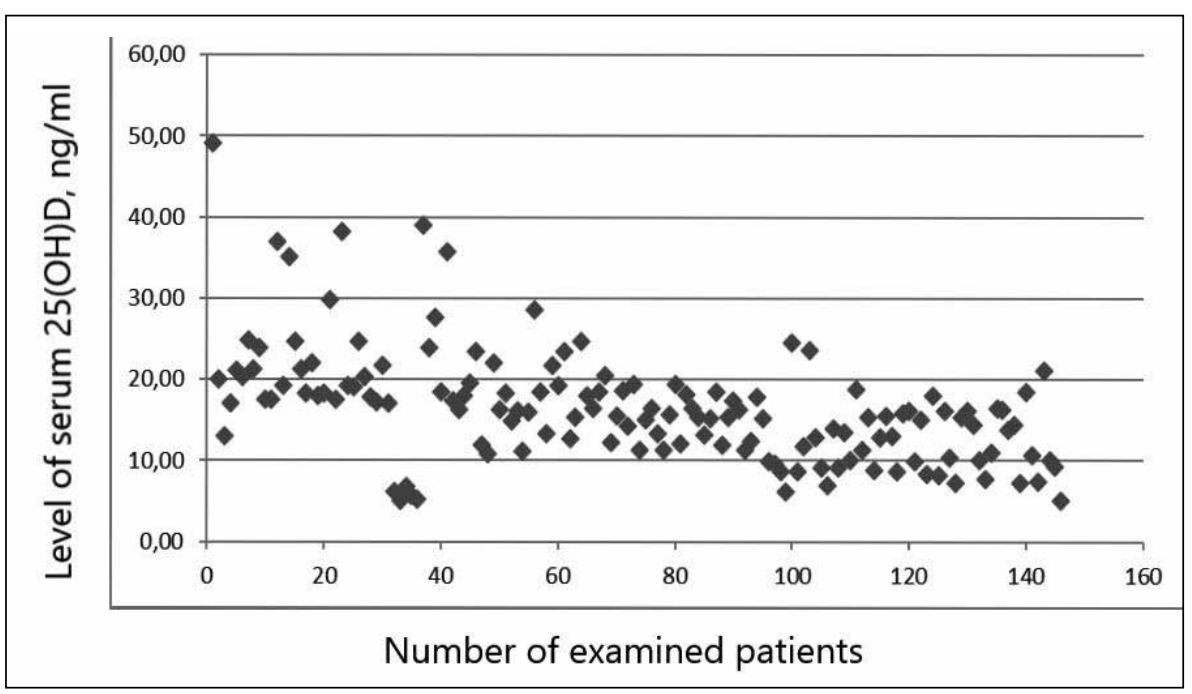

Fig. 1. Distribution of premenopausal women with autoimmune hypothyroidism according to the level of serum vitamin D.

Table I. Indicators of metabolic and hormonal disorders in premenopausal women with autoimmune hypothyroidism depending on the serum level of $25(\mathrm{OH})(\mathrm{M} \pm \mathrm{m})$.

\begin{tabular}{|c|c|c|c|c|c|}
\hline Characteristics & Group 1 & Group 2 & Group 3 & $\mathbf{p}_{1,2}$ & $\mathbf{p}_{1,3}$ \\
\hline Age (years) & $44,83 \pm 1,70$ & $46,16 \pm 0,69$ & $48,92 \pm 0,39$ & 0,043 & 0,014 \\
\hline BMI $\left(\mathrm{kg} / \mathrm{m}^{2}\right)$ & $21,28 \pm 1,10$ & $26,79 \pm 0,49$ & $30,33 \pm 0,273$ & 0,010 & 0,005 \\
\hline $25(\mathrm{OH}) \mathrm{D},(\mathrm{ng} / \mathrm{ml})$ & $39,02 \pm 2,09$ & $23,02 \pm 0,46$ & $13,84 \pm 0,38$ & 0,009 & 0,007 \\
\hline TSH (mIU/L) & $2,41 \pm 0,53$ & $2,58 \pm 0,36$ & $4,94 \pm 0,23$ & 0,007 & 0,005 \\
\hline fT3 (pmol/L) & $2,94 \pm 0,32$ & $2,60 \pm 0,19$ & $1,75 \pm 0,09$ & 0,004 & 0,002 \\
\hline fT4 (pmol/L) & $17,60 \pm 1,89$ & $15,21 \pm 0,73$ & $12,16 \pm 0,41$ & 0,015 & 0,008 \\
\hline TPOAb, (IU/ml) & $36,07 \pm 0,68$ & $46,93 \pm 1,07$ & $189,35 \pm 2,19$ & 0,022 & 0,043 \\
\hline $\mathrm{FBG}, \mathrm{mmol} / \mathrm{L}$ & $2,34 \pm 0,31$ & $2,37 \pm 0,11$ & $3,57 \pm 0,08$ & 0,008 & 0,002 \\
\hline Insulin $(\mu \mathrm{U} / \mathrm{mL})$ & $3,19 \pm 0,17$ & $4,43 \pm 0,21$ & $6,61 \pm 0,11$ & 0,005 & 0,002 \\
\hline HOMA-IR & $2,46 \pm 0,36$ & $2,66 \pm 0,14$ & $4,03 \pm 0,10$ & 0,003 & 0,002 \\
\hline $\mathrm{TC}, \mathrm{mmol} / \mathrm{L}$ & $1,66 \pm 0,09$ & $1,52 \pm 0,05$ & $1,32 \pm 0,02$ & 0,001 & 0,001 \\
\hline $\mathrm{TG}, \mathrm{mmol} / \mathrm{L}$ & $2,66 \pm 0,29$ & $2,79 \pm 0,15$ & $3,97 \pm 0,06$ & 0,003 & 0,001 \\
\hline $\mathrm{HDL} \mathrm{mmol} / \mathrm{L}$ & $0,94 \pm 0,09$ & $2,03 \pm 0,21$ & $4,19 \pm 0,12$ & 0,004 & 0,002 \\
\hline $\mathrm{LDL} \mathrm{mmol} / \mathrm{L}$ & $44,83 \pm 1,70$ & $46,16 \pm 0,69$ & $48,92 \pm 0,39$ & 0,043 & 0,014 \\
\hline$A C$ & $21,28 \pm 1,10$ & $26,79 \pm 0,49$ & $30,33 \pm 0,273$ & 0,010 & 0,005 \\
\hline
\end{tabular}

BMI: Body mass index; TSH: Thyroid-stimulating hormone; fT3: Free triiodothyonine; fT4: Free thyroxyne; TPOAb: Thyroid peroxidase antibodies; FBG: Fasting blood glucose; TC:Total cholesterol; TG: Triglycerides; HDL: High Density Lipoprotein; LDL: Low Density Lipoprotein; AC:Atherogenic coefficient. Notes: p1- the probability of difference in a group with sufficient vitamin D; p2 - the probability of difference with the indicator in the group with vitamin D insufficiency; $\mathrm{p} 3$ - the probability of difference with the indicator in the group with vitamin D deficiency.

Therefore, in women with autoimmune hypothyroidism and vitamin D deficiency were observed significant adverse disorders of carbohydrate and lipid metabolism which determines the degree of metabolic and cardiovascular risk.

Pearson correlation analysis was performed in the study groups to determine the relationship between vitamin D levels and anthropometric parameters, hormonal status, lipid and carbohydrate metabolism in premenopausal women with autoimmune hypothyroidism (Table II).

No correlation was found between level of $25(\mathrm{OH}) \mathrm{D}$ and age, $(p>0,05)$. A strong negative correlation was found between $25(\mathrm{OH}) \mathrm{D}$ and the level of TPOAb $(\mathrm{r}=-0,77)$ and with TSH $(r=-0,72)$. Positive correlations of mean strength were found with T3 $(r=0,43)$ and $\mathrm{T} 4(\mathrm{r}=0,44), \mathrm{p}<0,05$. Our results indicate that vitamin $\mathrm{D}$ deficiency is correlated with a decrease in thyroid function and the presence of TPOAb. In the analysis of the relationship of metabolic parameters was established a strong negative correlation between serum $25(\mathrm{OH}) \mathrm{D}$ concentration and BMI $(\mathrm{r}=-0,74)$, and TC level $(\mathrm{r}=-0,72)$; negative mean correlation was with AC $(r=-0,65)$, LDLc $(r=-0,58)$, HOMA-IR $(r=-0,57)$ and $\mathrm{TG}(\mathrm{r}=-0,46), \mathrm{p}<0,05$. The results of correlation analysis show that in premenopausal women with autoimmune hypothyroidism vitamin $\mathrm{D}$ level has linear relationships with anthropometric parameters and components of metabolic disorders which increases the risk of cardiometabolic complications. 
Table II. Correlations between 25 (OH) D level and hormonal, carbohydrate, lipid parameters in women with autoimmune hypothyroidism.

\begin{tabular}{ccc}
\hline Parameters & $\begin{array}{c}\text { Correlation coefficient } \\
\text { r (Pearson) }\end{array}$ & p \\
\hline Age (years) & $-0,30$ & 0,008 \\
\hline $\mathrm{BMI}\left(\mathrm{kg} / \mathrm{m}^{2}\right)$ & $-0,74$ & 0,009 \\
\hline $\mathrm{TSH}(\mathrm{mIU} / \mathrm{L})$ & $-0,72$ & 0,007 \\
\hline $\mathrm{fT3}(\mathrm{pmol} / \mathrm{L})$ & 0,46 & 0,006 \\
\hline $\mathrm{fT} 4(\mathrm{pmol} / \mathrm{L})$ & 0,44 & 0,009 \\
\hline $\mathrm{TPOAb},(\mathrm{IU} / \mathrm{ml})$ & $-0,72$ & 0,046 \\
\hline $\mathrm{TC}, \mathrm{mmol} / \mathrm{L}$ & $-0,72$ & 0,007 \\
\hline $\mathrm{TG}, \mathrm{mmol} / \mathrm{L}$ & $-0,46$ & 0,006 \\
\hline $\mathrm{HDL} \mathrm{mmol} / \mathrm{L}$ & 0,36 & 0,006 \\
\hline $\mathrm{LDL} \mathrm{mmol} / \mathrm{L}$ & $-0,58$ & 0,006 \\
\hline AC & $-0,65$ & 0,048 \\
\hline HOMA-IR & $-0,57$ & 0,007 \\
\hline
\end{tabular}

BMI: Body mass index; TSH: Thyroid-stimulating hormone; fT3: Free triiodothyonine; fT4: Free thyroxyne; TPOAb:Thyroid peroxidase antibodies; TC: Total cholesterol; TG: Triglycerides; HDL: High Density Lipoprotein; LDL: Low Density Lipoprotein; AC: Atherogenic coefficient.

\section{DISCUSSION}

Our findings do not contradict previous studies that have shown that the incidence of vitamin D hypovitaminosis in patients with autoimmune hypothyroidism is significantly higher in comparison with the healthy population. ChoonYoung Kim et al. [17] also investigated that low vitamin D status was significantly associated with autoimmune thyroid dysfunction, especially in premenopausal women. Dohee Kim [18] investigated the relationship between vitamin $\mathrm{D}$ deficiency and autoimmune thyroid disease, including hypothyroidism, and reported that lower 25 $(\mathrm{OH}) \mathrm{D}$ levels in serum were associated with higher TSH.

Comparative analysis of carbohydrate and lipid metabolism in the study groups showed a significant increase of their levels in vitamin D insufficiency and deficiency patients. In the group of women with vitamin D deficiency were higher $\mathrm{BMI}$, insulin resistance index HOMA and $\mathrm{AC}$ in comparison to women who had sufficient level of vitamin D. In the third group, in comparison with the first group, the level of LDLc was twice higher, and the $\mathrm{AC}$ was three times higher. Mean LDL cholesterol values were higher by $24.4 \%$, BMI by $42.5 \%$, HOMA insulin resistance index by $52.6 \%$.

It is known that impaired lipid metabolism plays an important role in the pathogenesis of cardiovascular diseases. Clinical studies have evaluated the mediate effect of vitamin D directly on cardiovascular function through regulation of lipid metabolism by cholecalciferol [19]. In addition, it is believed that the development of atherosclerosis begins at an early age, and its accelerated development underlies the increased risk of cardiovascular disease, which is associated with vitamin D deficiency [20]. Several studies have shown that subjects with an adequate serum concentration of $25(\mathrm{OH}) \mathrm{D}$ and high vitamin D intake have a more favorable lipid profile than those who have a vitamin $\mathrm{D}$ deficiency [21-23].

Our results indicate that vitamin D deficiency is correlated with a decrease in thyroid function and the presence of TPOAb. Similar results were obtained by the researchers Viktoria F. Koehler1 et al, who found an increase in the incidence of vitamin $\mathrm{D}$ deficiency in patients with autoimmune thyroid disease, especially with autoimmune thyroiditis [24].

The relationship between thyroid hormones, vitamin $\mathrm{D}$ and its metabolites remains unexplored. The available literature suggests that the relationship of $25(\mathrm{OH}) \mathrm{D}$ and thyroid hormones is complex and should be considered not only as a mechanism for regulating phosphorus-calcium homeostasis, but as a mechanism for cardiovascular pathology in patients with hypothyroidism and vitamin $\mathrm{D}$ deficiency. The need for additional administration and adequate doses of vitamin $\mathrm{D}$ to prevent cardiovascular disease in patients with autoimmune hypothyroidism remains insufficiently studied. Vitamin D deficiency screening is recommended for all patients with autoimmune thyroid disease, especially in premenopausal women.

\section{CONCLUSIONS}

1. In $78.8 \%$ of the surveyed premenopausal women with autoimmune hypothyroidism was determined vitamin $\mathrm{D}$ deficiency, in $17.1 \%$ insufficiency and in $4.1 \%$ sufficient level of vitamin D.

2. For autoimmune hypothyroidism accompanied by insufficiency and deficiency of vitamin D have been observed adverse disorders of carbohydrate and lipid metabolism: high negative correlation with BMI, TC; negative correlation of average strength with CA, HOMA resistance index, TG and LDL cholesterol, which determines the degree of metabolic disorders and possible cardiovascular risk in premenopausal women with autoimmune hypothyroidism.

3. It was determined that $25(\mathrm{OH}) \mathrm{D}$ had a high negative correlation between ATPO, TSH and average positive correlation with T3 and T4. Vitamin D deficiency is correlated with a decrease in thyroid function and severity of hypothyroidism, which determines the need for additional vitamin D prescription for all patients with autoimmune thyroid pathology.

\section{REFERENCES}

1. Batool S., Almaghaslah D., Motaal A.A. et al. Association of Autoimmune Hypothyroid Disease and Obesity with Vitamin D Deficiency in Female Patients.IntJPharmacol.2019;15(5):636-641.doi:10.3923/ijp.2019.636.641.

2. Charoenngam N.,Shirvani A., Holick M.F.The ongoing D-lemma ofVitamin D supplementation for nonskeletal health and bone health. Current Opinion in Endocrinology, Diabetes and Obesity.2019;26(6):301-305. doi.org/10.1097/MED.0000000000000508.

3. Rejnmark L., Bislev L.S., Cashman K.D. et al. Non-skeletal health effects of vitamin D supplementation: A systematic review on findings from meta-analyses summarizing trial data. PLOS ONE. 2017;12(7):1-39 doi. org/10.1371/journal.pone.0180512.

4. Povoroznyuk V.V., Pankiv I.V. Vitamin D levels in patients with autoimmune thyroiditis with diminished thyroid function. Int J Endocr. 2014;5(61):27-30. 
5. Yamamoto E.A., Jørgensen T.N. Relationships Between Vitamin D, Gut Microbiome, and Systemic Autoimmunity. Front Immunol. 2020;10:113. doi:10.3389/fimmu.2019.03141.

6. Spanier J.A., Nashold F.E., Nelson C.D. et al. Vitamin D3-mediated resistance to a multiple sclerosis model disease depends on myeloid cell 1,25-dihydroxyvitamin D3 synthesis and correlates with increased CD4+ T cell CTLA-4 expression. J Neuroimmunol. 2020;338:577105. doi:10.1016/j.jneuroim.2019.577105.

7. Harrison S.R., Danyang L., Jeffery L.E. et al. Vitamin D, Autoimmune Disease and Rheumatoid Arthritis. CalcifTissue Int. 2020;106(1):58-75. doi:10.1007/s00223-019-00577-2.

8. Ghaly S.Inflammatory bowel diseases: interrelationships between dietary vitamin $D$, exposure to UV radiation and the fecal microbiome, Expert Review of Gastroenterology \& Hepatology. 2019;13:1039-1048. doi: 10.1080/17474124.2019.1685874.

9. Choi Y.M., Kim W.G., Kim T.Y. et al. Low levels of serum vitamin D3 are associated with autoimmune thyroid disease in pre-menopausal women. Thyroid. 2014;24(4):655-661. doi:10.1089/thy.2013.0460.

10. Taylor P.N., Albrecht D., Scholz A. et al. Global epidemiology of hyperthyroidism and hypothyroidism. Nat Rev Endocrinol. 2018;14(5):301-316. doi:10.1038/nrend0.2018.18.

11. Wang J., Shishi L., Chen G. et al. Meta-analysis of the association between vitamin $D$ and autoimmune thyroid disease. Nutrients. 2015;7(4):2485-2498. doi:10.3390/nu7042485.

12. Shin D.Y., Kim K.J., Kim D. et al. Low serum vitamin D is associated with anti-thyroid peroxidase antibody in autoimmune thyroiditis. Yonsei Med J. 2014;55(2):476-481. doi:10.3349/ymj.2014.55.2.476.

13. Pan'kiv I.V. Intercommunication of vitamin D deficiency and autoimmune thyroid pathology. Buk Med Herald. 2015;4(76):132-136.

14. Choi Y.M., Kim W.G., Kim T.Y. et al. Low levels of serum vitamin D3 are associated with autoimmune thyroid disease in pre-menopausal women. Thyroid. 2014;24(4):655-661. doi:10.1089/thy.2013.0460.

15. Gurka M.J., Vishnu A., Santen R.J. et al. Progression of Metabolic Syndrome Severity During the Menopausal Transition. J Am Heart Assoc. 2016;5(8). doi:10.1161/JAHA.116.003609.

16. GuY., Wang Y., Zhang Q. et al. The association between thyroid function and incidence of metabolic syndrome in euthyroid subjects: Tianjin chronic low-grade systemic inflammation and health cohort study. Clin Endocrinol (Oxf). 2018;88(5):735-743. doi:10.1111/cen.13576.

17. Kim C.Y., Lee Y.J., Choi J.H. et al. The association between low Vitamin $D$ status and autoimmune thyroid disease in Korean premenopausal women:The 6th Korea national health and nutrition examination survey, 2013-2014. Korean J Fam Med. 2019;40(5):323-328. doi:10.4082/ kjfm.18.0075.

18. Kim D. Low vitamin D status is associated with hypothyroid hashimoto's thyroiditis. Hormones. 2016;15(3):385-393. doi:10.14310/ horm.2002.1681.

19. Saponaro F., Marcocci C., Zucchi R. Vitamin D status and cardiovascular outcome. J Endocrinol Invest. 2019;42(11):1285-1290. doi:10.1007/ s40618-019-01057-y.
20. Muñoz-Aguirre P., Denova-Gutiérrez E., Flores M. et al. High Vitamin $D$ consumption is inversely associated with cardiovascular disease risk in an urban Mexican population. PLoS One. 2016;11(11):1-13. doi:10.1371/journal.pone.0166869.

21. Salekzamani S., Mehralizadeh H., Ghezel A. et al. Effect of high-dose vitamin D supplementation on cardiometabolic risk factors in subjects with metabolic syndrome: a randomized controlled double-blind clinical trial. J Endocrinol Invest. 2016;39(11):1303-1313. doi:10.1007/s40618016-0507-8.

22. Glueck C.J., Jetty V., Rothschild M. et al. Associations between Serum 25-hydroxyvitamin D and Lipids, Lipoprotein Cholesterols, and Homocysteine. N Am J Med Sci. 2016;8(7):284-290. doi:10.4103/19472714.187137.

23. Dibaba D.T. Effect of vitamin D supplementation on serum lipid profiles: a systematic review and meta-analysis. Nutr Rev. 2019;77(12):890-902. doi:10.1093/nutrit/nuz037.

24. Koehler V.F., Filmann N., Mann W.A. Vitamin D Status and Thyroid Autoantibodies in Autoimmune Thyroiditis. Horm Metab Res. 2019;51(12):792-797. doi:10.1055/a-1023-4181.

\section{ORCID and contributionship:}

Oksana O. Chukur: 0000-0002-9371-2220 A,B,D

Nadiya V. Pasyechko: 0000-0002-2081-4269 A,E

Anzhela O. Bob: 0000-0003-1435-8233 B,D,F

Iryna V. Smachylo: 0000-0003-4323-8628 C,E

Liudmyla V. Radetska: 0000-0002-2916-1831 E,F

\section{Conflict of interest:}

The Authors declare no conflict of interest.

\section{CORRESPONDING AUTHOR Oksana 0. Chukur}

I. Horbachevsky Ternopil National Medical University

1 Maidan Voly st., 46002 Ternopil, Ukraine;

tel: +380978218809

e-mail:chukur@tdmu.edu.ua

Received: 2020-05-13

Accepted: 2021-06-01

A - Work concept and design, B - Data collection and analysis, C - Responsibility for statistical analysis, D-Writing the article, $\mathbf{E}$-Critical review, $\mathbf{F}$ - Final approval of the article 